\title{
Observation of incomplete fusion at low angular momenta
}

\author{
Devendra P. Singh ${ }^{1, a}$, Abhishek Yadav ${ }^{2}$, Indu Bala², Anubhav Raghav ${ }^{3}$, Mohd. Shuaib ${ }^{3}$, Prabhat Kumar ${ }^{3}$, Pushpen- \\ dra P. Singh ${ }^{4}$, Unnati ${ }^{3}$, M. K. Sharma ${ }^{5}$, Vijay R. Sharma ${ }^{3}$, R. Kumar ${ }^{2}$, R. K. Gupta ${ }^{1}$, B. P. Singh ${ }^{3}$, and R. Prasad ${ }^{3}$ \\ ${ }^{1}$ Department of Physics, University of Petroleum and Energy Studies, Dehradunn-248 007, Uttrakhand, India \\ ${ }^{2}$ NP Group: Inter-University Accelerator Centre, Aruna Asaf Ali Marg, New Delhi-110 067, Delhi, India \\ ${ }^{3}$ Department of Physics, Aligarh Muslim University, Aligarh-202 002, Uttar Pradesh, India \\ ${ }^{4}$ Department of Physics, Indian Institute of Technology Ropar, Roopnagar-140 001, Punjab, India \\ ${ }^{5}$ Department of Physics, S. V. College, Aligarh-202 001, Uttar Pradesh, India
}

\begin{abstract}
Present work deals with experimental studies of incomplete fusion reaction dynamics using off-line $\gamma$-ray spectrometry at energies as low as $\approx 3-6 \mathrm{MeV} /$ nucleon. Excitation functions for five reaction products populated via complete and/or incomplete fusion processes in ${ }^{16} \mathrm{O}+{ }^{130} \mathrm{Te}$ system have been measured and compared with the predictions of the statistical model code PACE4. A significant enhancement in the measured excitation functions compared to theoretical predictions for $\alpha$-emitting channels has been observed and is attributed to incomplete fusion processes. The relative strength of incomplete fusion has been found to increase with projectile energy. Results show that incomplete fusion is associated even for angular momenta lesser than the critical angular momentum for complete fusion and also reveals importance of incomplete fusion even at energies as low as $\approx 3-6 \mathrm{MeV} /$ nucleon.
\end{abstract}

\section{Introduction}

In recent years, incomplete fusion (ICF) processes in heavy-ion interactions around the Coulomb barrier have been a topic of interest for exploring the nuclear structure and reaction dynamics [1-6]. In the ICF processes, direct $\alpha$-particles have been observed in the forward cone with nearly the same velocity as that of the incident ion [7]. It may be pointed out that, at 3-6 MeV/nucleon complete fusion $(\mathrm{CF})$ is supposed to be the sole contributor to the total fusion cross-section $[2,8]$. Interestingly, $\alpha$-emitting channels at energies $\approx 3-6 \mathrm{MeV} /$ nucleon are found to show enhancement of cross-sections over the statistical model predictions, which may be due to the projectile breakup processes in these reactions. At higher projectile energies ( $\approx 10 \mathrm{MeV} /$ nucleon), Wilczynski et al. [9] have well explained the cross-sections for ICF reactions based on partial statistical equilibrium and on the idea of a generalized concept of angular momentum. However, on the basis of the above prescription, ICF reactions could not be explained at lower projectile energies, where the maximum angular momentum values $\left(\ell_{\max }\right)$ are less than the critical angular momentum $\left(\ell_{\text {critical }}\right)$. The $\gamma$-multiplicity measurements by Wilczynski et al. [9], Inamura et al. [10], Gerschel et al. [11], and Trautmann et al. [12] also indicate that such breakup fusion, in general, involves $\ell \geq \ell_{\text {critical }}$. However, studies [13] on spherical targets showed involvement of $\ell$-values in ICF lower than $\ell_{\text {critical }}$ as well, giving rise to conflicting reports on the

\footnotetext{
a e-mail: dpsingh19@gmail.com
}

dependence of ICF on the angular momentum. Further, it may be pointed out that the available theoretical models [9, 14-17] satisfactorily predict the magnitude of ICF contribution, to some extent, in some cases at energies $\approx 10 \mathrm{MeV} /$ nucleon, but none of these models is able to successfully explain such data at low energies. In view of the above, a clear picture of the mechanism of ICF has yet to emerge, particularly at energies $\approx 3-5 \mathrm{MeV} /$ nucleon.

\section{Experiments}

A beam of ${ }^{16} \mathrm{O}^{7+}$ obtained from the 15-UD Pelletron accelerator of the Inter-University Accelerator Centre, New Delhi, India, has been used to irradiate ${ }^{130} \mathrm{Te}$ target samples (enrichment $\approx 61 \%$ ), prepared using vacuum evaporation on $\mathrm{Al}$ foils of thickness $\approx 6.75 \mathrm{mg} / \mathrm{cm}^{2}$. The thickness of sample deposition $\left(\approx 1.8 \mathrm{mg} / \mathrm{cm}^{2}\right)$ in each target was determined by the $\alpha$-transmission method. Two stacks, containing two ${ }^{130} \mathrm{Te}$ samples each, followed by $\mathrm{Al}$ catchers, were irradiated at 85 and $90 \mathrm{MeV}$, respectively, in the General Purpose Scattering Chamber (having in-vacuum transfer facility) with a constant beam current $(\approx 3 \mathrm{pnA})$ for $\approx 8 \mathrm{~h}$ duration. The irradiated samples along with the catchers were taken to a high-purity Ge detector for $\gamma$ counting. The resolution of the detector system was $\approx 2 \mathrm{keV} \mathrm{FWHM} \mathrm{for} \mathrm{the} 1332.0 \mathrm{keV} \gamma$-line of ${ }^{60} \mathrm{Co}$. The reaction residues of interest have been identified by their measured half-lives $\left(\mathrm{T}_{1 / 2}\right)$ and characteristic $\gamma$-ray energies. A critical evaluation of the uncertainties in the 
measured cross-sections has been considered. The errors in the measured production cross sections may arise due to (i) the nonuniformity of target foils, (ii) fluctuations in the beam current, (iii) the uncertainty in geometry dependent efficiency of HPGe detector, and (iv) due to the dead time of the spectrometer. Detailed discussion on the error analysis is given elsewhere [4-6]. The overall errors including statistical errors are estimated to be $\geq 15 \%$, excluding the uncertainty in branching ratio, decay constant, etc.

\section{Measurements and analysis}

The cross-sections for $\left.(5 n))^{141} \mathrm{Nd}, \quad(\alpha 3 n)\right)^{139} \mathrm{Ce}$, $(3 \alpha \mathrm{n})^{133} \mathrm{Xe}^{m},(3 \alpha \mathrm{n})^{133} \mathrm{Xe}^{g}$, and $(3 \alpha 3 n){ }^{131} \mathrm{Xe}^{m}$ residues have been measured in the energy range of $\approx 60-90 \mathrm{MeV}$. In the present work, $5 \mathrm{n}$ channel residues $\left[{ }^{141} \mathrm{Nd}\left(\mathrm{t}_{1 / 2}=\right.\right.$ $2.49 \mathrm{~h}$ )] could be identified by the characteristic $\gamma$-ray of $1127 \mathrm{keV}$ and also by measuring its half-life using decay curve analysis. Further, the ${ }^{139} \mathrm{Ce}$ residues may be formed by the $\mathrm{CF}$ of ${ }^{16} \mathrm{O}$ and ${ }^{130} \mathrm{Te}$, forming the composite system ${ }^{146} \mathrm{Nd}^{*}$, which may decay by the evaporation of an $\alpha$-particle and three neutrons. ${ }^{139} \mathrm{Ce}$ residues may also be produced if the fragment ${ }^{12} \mathrm{C}$ (if ${ }^{16} \mathrm{O}$ undergoes breakup into an $\alpha$ particle and ${ }^{12} \mathrm{C}$ fragments) fuses with the ${ }^{130} \mathrm{Te}$ target nucleus followed by the evaporation of three neutrons. In the same way, ${ }^{133} \mathrm{Xe}^{g, m}$ and ${ }^{131} \mathrm{Xe}^{m}$ residues may also be formed by $\mathrm{CF}$ as well as ICF processes. Theoretical calculations of cross-sections for the residues populated via $\mathrm{CF}$ and/or ICF channels have also been done using the code PACE4 [18]. This model follows the correct procedure for angular momentum coupling at each stage of de-excitation. The angular momentum conservation is explicitly taken into account at each step. In this code the fusion cross-section was calculated using the Bass formula. The details of this model are given in our earlier work [4]. In this model the most important parameter is level density parameter (LDP). The LDP (a $=\mathrm{A} / \mathrm{K})$, where $\mathrm{A}$ is the atomic mass of the compound nucleus $(\mathrm{CN})$ and $\mathrm{K}$ is a free parameter. It is not out of place to mentioned that in this code ICF of the incident ion is not taken into consideration, so any enhancement in the measured cross-section compared to PACE4 code predictions may be attributed to breakup fusion channels. The $\alpha 3 \mathrm{n}$ channel gives rise to the population of ${ }^{139} \mathrm{Ce}$. The isomeric state of ${ }^{139} \mathrm{Ce}\left(\mathrm{t}_{1 / 2} \approx 56 \mathrm{~s}\right)$ decays completely via transition to the ground state of ${ }^{139} \mathrm{Ce}$. Therefore, the measured activity of ${ }^{139} \mathrm{Ce}^{g}$ may be taken as the sum of the metastable and ground states of ${ }^{139} \mathrm{Ce}$. In order to determine the ICF contribution to the measured $\alpha$-emitting channels, the measured $\Sigma \sigma_{\text {exp }}(\alpha)$ (sum of cross-sections of all measured $\alpha$-emitting channels) has been compared with the corresponding calculated values based on $\mathrm{CF}$ calculations, i.e., $\Sigma \sigma_{T h}(\alpha)$. In Fig. 1(a), a comparison of $\Sigma \sigma_{\text {exp }}(\alpha)$ has been made with corresponding $\Sigma \sigma_{T h}(\alpha)$ calculated using PACE4. It may be observed from Fig. 1(a) that the $\Sigma \sigma_{T h}(\alpha)$ obtained from PACE4 predictions are significantly lower than the $\Sigma \sigma_{\text {exp }}(\alpha)$ in the entire energy
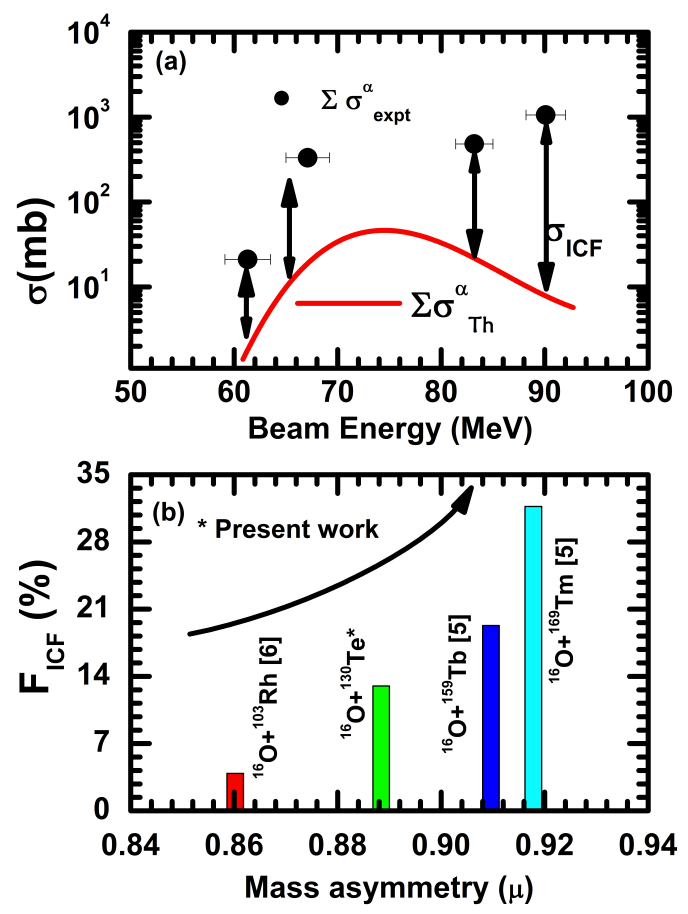

Figure 1. (a) Comparison of the sum of the measured crosssections for $\alpha$-emitting channels and calculated values. The increasing difference between the experimental and calculated values with energy indicates the dominance of ICF processes with energy. (b) The ICF fraction $\left(\mathrm{F}_{I C F}\right)$ as a function of mass asymmetry at a constant relative velocity $(\beta=0.055 \mathrm{c})$ for the presently studied system along with results from the literature $[5,6]$.

range. The enhancement of the experimental values compared to the theoretical predictions may be due to the ICF processes and has been denoted by $\sigma_{I C F}$. It may also be noted that the difference between $\Sigma \sigma_{\text {exp }}(\alpha)$ and $\Sigma \sigma_{T h}(\alpha)$ increases with energy throughout the entire energy region of interest, indicating the dominance of ICF with a maximum ICF contribution at the highest studied energy.

Further, the isomeric cross-section ratios (ICRs) for the residues in the reaction ${ }^{130} \mathrm{Te}\left({ }^{16} \mathrm{O}, 3 \alpha \mathrm{n}\right){ }^{133} \mathrm{Xe}$ produced via $\mathrm{CF}$ and/or ICF channels are also found to increase with energy, in general. Since the ICF reactions are considered to take place in peripheral collisions, a relatively large amount of angular momentum may be transferred and this may increase with energy. The increase in isomeric population with energy shows that a part of the input angular momentum may get converted to the nuclear spin and the isomeric population may increase. Thus, the ICR may depend strongly on the relative spins of metastable and ground states and also on the energy difference between the levels. In order to understand how the ICF fraction varies with the entrance channel mass asymmetry $(\mu)$, the value of $\mathrm{F}_{I C F}(\%)$ for the ${ }^{16} \mathrm{O}+{ }^{130} \mathrm{Te}$ system has been compared with those found in the literature $[9,10]$ at a constant relative velocity $(\beta=0.055 \mathrm{c})$. As can be seen from Fig. 1(b) the $\mathrm{F}_{I C F}$ is found to increase with mass asymmetry $(\mu)$ for the ${ }^{16} \mathrm{O}$ projectile with different 


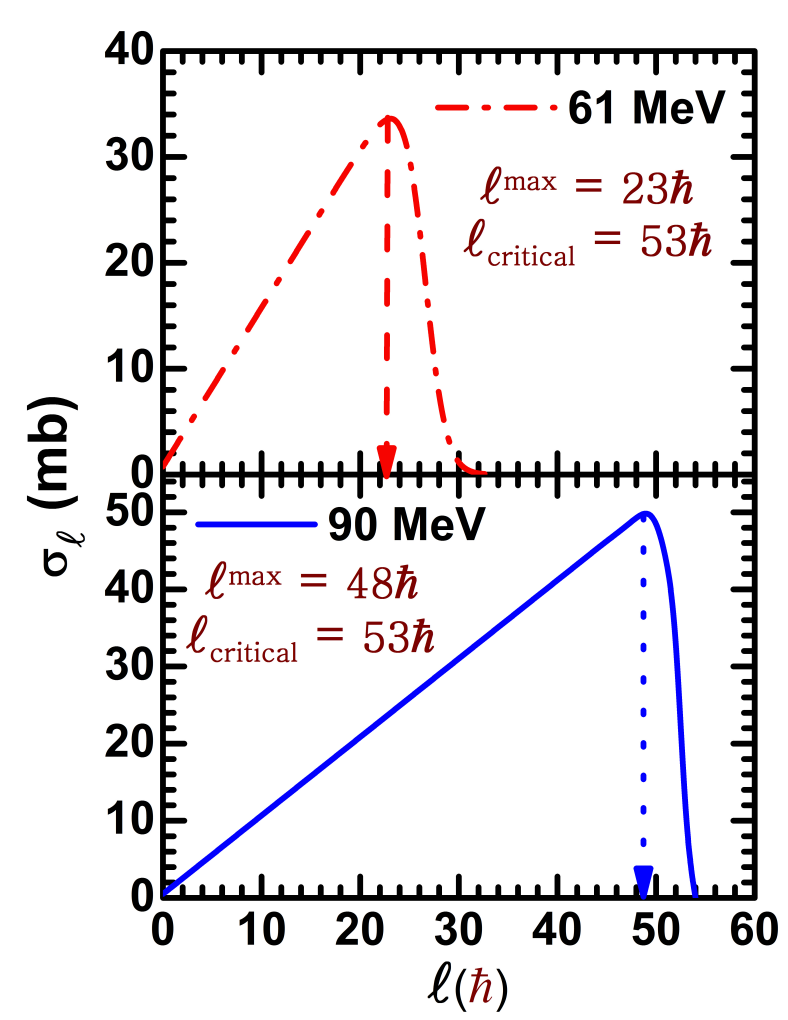

Figure 2. Typical Fusion $\ell$-distributions calculated by using the code CCFULL for the ${ }^{16} \mathrm{O}+{ }^{130} \mathrm{Te}$ system at $\mathrm{E}_{l a b} \approx 61$ and 90 $\mathrm{MeV}$.

targets. As inferred from this figure, the ICF probability is higher for more mass-asymmetric systems, which is in accordance with the Morgenstern mass-asymmetry systematics [19].

Further, it is possible to calculate the cross-sections for CF and ICF channels separately using the 'sumrule' model [20,21], based on the idea of a generalized concept of critical angular momentum following partial statistical equilibrium. The underlying assumption in the 'sumrule' model is that the ICF channels open up only for those partial waves which have $\ell$ values greater than $\ell_{\text {critical }}$ (i.e., $\ell \geq \ell_{\text {critical }}$ ). On the other hand, partial waves with $\ell$ $\leq \ell_{\text {critical }}$ contribute to CF. The present findings indicate that a diffused boundary in $\ell$ space may penetrate close to the barrier, such that fusion may take place even for $\ell<$ $\ell_{\text {critical }}$. In order to ascertain the above in the $\ell$ distribution for the ${ }^{16} \mathrm{O}+{ }^{130} \mathrm{Te}$ system, the $\ell_{\text {critical }}$ value has been calculated [9] and is found to be 53h. Fig. 2 shows the fusion $\ell$ distributions for the ${ }^{16} \mathrm{O}+{ }^{130} \mathrm{Te}$ system calculated using the code CCFULL [22] at two extreme energies 61 and $90 \mathrm{MeV}$, respectively. The values of $\ell_{\max }$ at two energies (61 and $90 \mathrm{MeV}$ ) are found to be $\approx 23 \hbar$ and $48 \hbar$, respectively which are less than the $\ell_{\text {critical }}$ value $(53 \hbar)$ for fusion for this system. From Fig. 2, it may also be seen that, even at the highest studied energy, the maxima of $\ell$ values are not as high as $\ell_{\text {critical }}$ for fusion for both the studied energies. Thus, the ICF contributions are expected to be negligible at these energies. However, the present measurements for ICF channels suggest that a significant number of partial waves below $\ell_{\text {critical }}$ may contribute to ICF channels. The present observations clearly indicate a diffused boundary for $\ell$-values, contrary to the sharp cut-off model, that may penetrate close to the barrier.

\section{Summary}

In the present work, for $\alpha$-emitting channels, enhancement in cross-sections over the predictions of statistical model calculations has been observed and may be attributed to the prompt break-up of the projectile, leading to ICF processes. A comparison of data for ICF contribution for the same projectile with different targets indicates a strong and increasing trend of incomplete fusion fraction with target mass number and showing a strong mass asymmetry dependence. The 'sumrule' model calculations highly underestimate the ICF cross-sections, indicating the limitation of the model assumption that a substantial contribution to ICF comes from collision trajectories with $\ell>\ell_{\text {critical }}$. In the energy range of the present study, calculations indicate that $\ell_{\max }$ is less than $\ell_{\text {critical }}$, thus, significant cross-sections for ICF channels at these beam energies indicate the contribution from collision trajectories with $\ell<\ell_{\text {critical }}$ as well.

\section{Acknowledgements}

The authors thank the Director of IUAC, New Delhi, India, and to the Dean, CoES, UPES, Dehradun for providing facilities to carry out the work. RP and BPS thank to UGC and DST, New Delhi, India, for providing financial support. DPS and AY thank to the DST for providing financial support through Project No. SR/FTP/PS-025/2011 and SB/FTP/PS-194/2013, respectively, under the Fast Track Scheme for Young Scientists.

\section{References}

[1] P. R. S. Gomes et al., Phys. Rev. C 73 (2006); Phys. Lett. B 601, 20 (2004).

[2] M. Das Gupta et al., Nucl. Phys. A 787, 144 (2007); Phys. Rev. C 70, 024606 (2004).

[3] Abhishek Yadav et al., Phys. Rev. C 85, 034614 (2012); ibid 85, 064617 (2012); ibid 86, 014603 (2012) and references therein.

[4] D. P. Singh et al., Phys. Rev. C 80, 014601 (2009) ; Phys. Rev. C 81, 054607 (2010).

[5] P. P. Singh et al., Phys. Rev. C 77, 014607 (2008) ; Phys. Lett. B 671, 20 (2009).

[6] Unnati et al., Nucl. Phys. A 811, 77 (2008).

[7] H. C. Britt and A. R. Quinton, Phys. Rev. C 124, 877 (1961). 
[8] L. F. Canto et al., Phys. Rev. C 58, 1107 (1998).

[9] J. Wilczynski et al., Phys. Rev. Lett. 45, 606 (1980).

[10] T. Inamura et al., Phys. Lett. B 68, 51 (1977) and Phys. Lett. B 84, 71 (1979).

[11] C. Gerschel et al., Nucl. Phys. A 387, 297 (1982).

[12] W. Trautmann et al., Phys. Rev. Lett. 53, 1630 (1984).

[13] I. Tserruya et al., Phys. Rev. Lett. 60, 14 (1988).

[14] T. Udagawa and T. Tamura, Phys. Rev. Lett. 45, 1311 (1980).

[15] J. P. Bondrof et al., Nucl. Phys. A 333, 285 (1980).
[16] R. Weiner et al., Nucl. Phys. A 286, 282 (1977).

[17] V. I. Zagrebaev et al., Ann. Phys. (NY) 197, 33 (1990).

[18] O. B. Tarasov and D. Bazin, Nucl. Instrum. Methods Phys. Res., Sect. B 204, 174 (2003).

[19] H. Mogenstern et al., Phys. Lett. B 113, 463 (1982); Z. Phys. A 324, 443 (1986).

[20] J. Wilczynski et al., Nucl. Phys. A 373, 109 (1982).

[21] K. Siwek-Wilczynska et al., Phys. Rev. Lett. 42, 1599 (1979).

[22] K. Hagino et al., Comput. Phys. Commun. 123, 143 (1999). 